\title{
A STUDY OF COUSIN COMPLEXES THROUGH THE DUALIZING COMPLEXES
}

\author{
MOHAMMAD T. DIBAEI
}

\begin{abstract}
For the Cousin complex of certain modules, we investigate finiteness of cohomology modules, local duality property and injectivity of its terms. The existence of canonical modules of Noetherian non-local rings and the Cousin complexes of them with respect to the height filtration are discussed .
\end{abstract}

\section{INTRODUCTION}

This Paper is the continuation of [DT1] and [DT2]. We have seen in [DT2] that if $M$ is a finitely generated module over a local ring $A$ which possesses the fundamental dualizing complex $I^{\bullet}$, then, under certain conditions on $M, \operatorname{Hom}_{A}\left(M, I^{\bullet}\right)$ represents the Cousin complex of the module $H^{\operatorname{dim} A-\operatorname{dim}_{A}(M)}\left(\operatorname{Hom}_{A}\left(M, I^{\bullet}\right)\right)$, the $\left(\operatorname{dim} A-\operatorname{dim}_{A}(M)\right)$-th cohomology module of the complex $\operatorname{Hom}_{A}\left(\mathrm{M}, I^{\bullet}\right)$, with respect to an appropriate filtration of $\operatorname{Spec}(A)$; and that we can reconstruct the Cousin complex of the module $M$ by means of the fundamental dualizing complex (see the proof of [DT2, Lemma 3.1]). In section 2, we pursue our expectation that the Cousin complexes of such modules will inherit some properties of the dualizing complex of the ring itself. We will show that if $(A, \mathfrak{m})$ is a Noetherian local ring (not necessarily possessing a dualizing complex) such that all of its formal fibres are Cohen-Macaulay rings, $M$ is a finitely generated $A$-module which satisfies the condition $\left(S_{2}\right)$ of Serre and $\operatorname{Min}_{\widehat{A}}(\widehat{M})=\operatorname{Ass}_{\widehat{A}}(\widehat{M})$, then all the cohomology modules of $C_{A}(M)$, the Cousin complex of $M$ with respect to the $M$-height filtration, are finitely generated $A$ modules (a result proved also, under different hypotheses, by T. Kawasaki in [K, Theorem 1.1]), and also they satisfy a local duality property which is analogous to that of the Grothendieck local duality. Here, $\widehat{M}$ denotes the completion of $M$ with respect to the $\mathfrak{m}$-adic topology. We present, in section 3, a number of applications

2000 Mathematics Subject Classification. 13D25; 13H10; 13D45 .

Key words and phrases. Cousin complexes, dualizing complexes, Gorenstein modules.

This research is supported in part by MIM Grant P82-104.

Address: Mosaheb Institute of Mathematics, Teacher Training University, 599 Taleghani Avenue, 19165 Tehran, Iran.

E-mail address: dibaeimt@ipm.ir . 
which come out of these results and those of [DT1] and [DT2] .

In the remainder of the paper we study the Cousin complex of certain modules over Noetherian non-local ring $R$. In section 4 we recall the notion of canonical modules for such a ring $R$ and prove the existence of them when $R$ possesses a dualizing complex and satisfies $\left(S_{2}\right)$. As a result we present a partial generalization of [BH, Proposition 3.3.18]. In section 5, we generalize [DT1, Corollary 3.4] for non-local case and give a characterization for the Cousin complex of a canonical module w.r.t. the height filtration to be a dualizing complex. Finally, we give an explicit description for all indecomposables injective modules which improves [DT1, Corollary 3.3 ] .

\section{Preliminaries}

Throughout $A$ is a Noetherian local ring of dimension $d$ with the maximal ideal $\mathfrak{m}$, and $M$ is a finitely generated $A$-module of $\operatorname{dim}_{A}(M)=s$. A finitely generated $A$-module $K_{M}$ (if it exists) is called the canonical module of $M$ if $K_{M} \otimes_{A} \widehat{A} \cong$ $\operatorname{Hom}_{A}\left(H_{\mathfrak{m}}^{s}(M), E(A / \mathfrak{m})\right)$, where $H_{\mathfrak{m}}^{s}(M)$ is the $\mathrm{s}$-th local cohomology module of $M$ w.r.t. $\mathfrak{m}$, and $E(A / \mathfrak{p})$ is the injective envelope of the $A$-module $A / \mathfrak{p}$ with $\mathfrak{p} \in$ $\operatorname{Spec}(A)$. The canonical module of $M$ (if exists) is unique up to isomorphism (see [HK, Lemma 5.8] ).

1.1. Some remarks. If $A$ possesses a dualizing complex, then it possesses the fundamental dualizing complex

$$
I^{\bullet}: 0 \longrightarrow I^{0} \stackrel{\delta^{0}}{\longrightarrow} I^{1} \stackrel{\delta^{1}}{\longrightarrow} \cdots \stackrel{\delta^{d-1}}{\longrightarrow} I^{d} \longrightarrow 0
$$

which we call " the dualizing complex" (see $[\mathrm{H}]$ ), with the following properties :

(i) for each $i \geq 0, H^{i}\left(I^{\bullet}\right)$, the $i$-th cohomology module of $I^{\bullet}$, is finitely generated.

(ii) $I^{i}=\bigoplus E(A / \mathfrak{p}), \quad i=0,1, \ldots, d$. If $A$ possesses the dualizing complex $I^{\bullet}$, then the module $K_{M}:=H^{d-s}\left(\operatorname{Hom}_{A}\left(M, I^{\bullet}\right)\right)$ is the canonical module of $M$. If $K_{M}$ is the canonical module of $M$, it is easy to see that $\widehat{\left(K_{M}\right)} \cong K_{\widehat{M}}$ is the canonical module of $\widehat{M}$, as $\widehat{A}$-module. For the module $M$, we set $\operatorname{Min}_{A}(M)$ to denote the set of all minimal elements of $\operatorname{Supp}_{A}(M)$, and

$$
\operatorname{Assh}_{A}(M)=\left\{\mathfrak{p} \in \operatorname{Supp}_{A}(M): \operatorname{dim}_{A}(A / \mathfrak{p})=\operatorname{dim}_{A}(M)\right\} .
$$

Also $M$ is said to satisfy $\left(S_{n}\right)$ if $\operatorname{depth}_{A_{\mathfrak{p}}}\left(M_{\mathfrak{p}}\right) \geq \min \left\{n, \mathrm{ht}_{M}(\mathfrak{p})\right\}$ for all $\mathfrak{p} \in$ $\operatorname{Supp}_{A}(M)$. A filtration of $\operatorname{Spec}(A)$ is a descending sequence $\mathcal{F}=\left(F_{i}\right)_{i \geq 0}$ of subsets of $\operatorname{Spec}(A)$, so that,

$$
F_{0} \supseteq F_{1} \supseteq \cdots \supseteq F_{i} \supseteq \cdots
$$


with the property that, for each $i \geq 0$, each member of $\partial F_{i}=F_{i}-F_{i+1}$ is a minimal member of $F_{i}$, with respect to inclusion. We say that $\mathcal{F}$ admits $M$ if $\operatorname{Supp}_{A}(M) \subseteq F_{0}$. Suppose that $\mathcal{F}$ is a filtration of $\operatorname{Spec}(A)$ that admits $M$. The Cousin complex $C(\mathcal{F}, M)$ for $M$ with respect to $\mathcal{F}$ has the form

$$
0 \stackrel{d^{-2}}{\longrightarrow} M \stackrel{d^{-1}}{\longrightarrow} M^{0} \stackrel{d^{0}}{\longrightarrow} M^{1} \longrightarrow \cdots \longrightarrow M^{n-1} \stackrel{d^{n-1}}{\longrightarrow} M^{n} \longrightarrow \cdots
$$

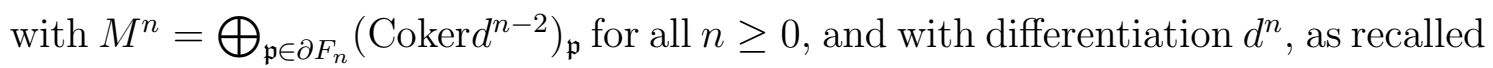
in $[\mathrm{T}]$.

Set $\mathcal{H}_{M}=\left(H_{i}\right)_{i \geq 0}$ to be the $M$-height filtration of $\operatorname{Spec}(A)$, i.e. $H_{i}=\{\mathfrak{p} \in$ $\left.\operatorname{Supp}_{A}(M): \operatorname{ht}_{M}(\mathfrak{p}) \geq i\right\}$. We denote the Cousin complex of $M$ with respect to $\mathcal{H}_{M}$ by $C_{A}(M)$.

Set $\bar{A}=A / 0:_{A} M$. Then $M$ has a natural structure as $\bar{A}$-module. It is straightforward to see that each term of the complex $C_{A}(M)$ has a natural $\bar{A}$-module structure and each differentiation of $C_{A}(M)$ is an $\bar{A}$-homomorphism. Moreover, it is straightforward to see that :

1.2. Lemma. If $M$ is a finitely generated $A$-module and $\bar{A}:=A / 0:_{A} M$, then there exists an isomorphism of complexes $C_{A}(M) \cong C_{\bar{A}}(M)$.

The following lemma will be used later.

1.3. Lemma. [P, Theorem 3.5] Suppose that all formal fibres of A are CohenMacaulay. If $M$ is a finitely generated A-module, then there is a morphism of complexes $u^{\bullet}: C_{A}(M) \bigotimes_{A} \widehat{A} \longrightarrow C_{\widehat{A}}(\widehat{M})$ which is a monomorphism. Moreover the quotient complex $Q^{\bullet}$, in the exact sequence

$$
0 \longrightarrow C_{A}(M) \bigotimes_{A} \widehat{A} \stackrel{u^{\bullet}}{\longrightarrow} C_{\widehat{A}}(\widehat{M}) \longrightarrow Q^{\bullet} \longrightarrow 0,
$$

is an exact complex, so that, for each $i \geq 0$, there exists an $\widehat{A}$-isomorphism $H^{i}\left(C_{A}(M)\right) \bigotimes_{A} \widehat{A} \cong H^{i}\left(C_{\widehat{A}}(\widehat{M})\right)$.

1.4. Convention. For a complex $C^{\bullet}: 0 \longrightarrow C^{-1} \stackrel{\theta^{-1}}{\longrightarrow} C^{0} \stackrel{\theta^{0}}{\longrightarrow} C^{1} \stackrel{\theta^{1}}{\longrightarrow} \cdots$ of $A$ -module and $A$-homomorphisms, we denote

$$
C^{\prime}: 0 \longrightarrow C^{0} \stackrel{\theta^{0}}{\longrightarrow} C^{1} \stackrel{\theta^{1}}{\longrightarrow} \cdots \text { and }\left(C^{\prime}\right)^{*}: 0 \longrightarrow H^{0}\left(C^{\prime}\right) \longrightarrow C^{0} \longrightarrow C^{1} \longrightarrow \cdots \text {. }
$$

\section{Some properties of Cousin COMplexes}

In this section we establish some properties of certain complexes by means of dualizing complexes. First we show that these Cousin complexes have finitely generated cohomologies. 
2.1. Theorem. Let $A$ be a ring with Cohen-Macaulay formal fibres. Assume that $M$ satisfies $\left(S_{2}\right)$ and $\operatorname{Min}_{\widehat{A}}(\widehat{M})=\operatorname{Assh}_{\widehat{A}}(\widehat{M})$. Then $C_{A}(M)^{\prime}$ has finitely generated cohomology modules.

Proof. Since $M$ satisfies $\left(S_{2}\right)$, the Cousin complex $C_{A}(M)$ is exact at $M$ and $M^{0}$ (see [SSc, Example 4.4]). Thus $H^{0}\left(C_{A}(M)^{\prime}\right)=M$. So it is enough to prove that $H^{i}\left(C_{A}(M)^{\prime}\right)$ is finitely generated for all $i>0$. Note that, for $i>0$, we have $H^{i}\left(C_{A}(M)^{\prime}\right)=H^{i}\left(C_{A}(M)\right)$. By 1.3, we have $H^{i}\left(C_{A}(M)\right) \bigotimes_{A} \widehat{A} \cong H^{i}\left(C_{\widehat{A}}(\widehat{M})\right)$. Therefore $C_{\widehat{A}}(\widehat{M})$ is also exact at $\widehat{M}$ and $(\widehat{M})^{0}$; so that $\widehat{M}$ satisfies $\left(S_{2}\right)$ as $\widehat{A}-$ module. Since $\operatorname{Min}_{\widehat{A}}(\widehat{M})=\operatorname{Assh}_{\widehat{A}}(\widehat{M})$, by [DT2, Theorem 3.2], all cohomology modules $H^{i}\left(C_{\widehat{A}}(\widehat{M})\right)$ are finitely generated $\widehat{A}$-module. Now, by [M, Exercise 7.3], the claim follows.

2.2. Corollary. Assume that the ring $A$ satisfies $\left(S_{2}\right)$ and all formal fibres of $A$ are Cohen-Macaulay. Then $C_{A}(A)$, the Cousin complex of $A$, has finitely generated cohomology modules.

Proof. By [M, Theorem 23.9], $\widehat{A}$ satisfies $\left(S_{2}\right)$ and thus $\operatorname{Min}(\widehat{A})=\operatorname{Assh}(\widehat{A})(\operatorname{see}[\mathrm{DT} 1$, Remark 1.3]).

For a ring $A$ and a property $P$, the $P$ locus of $A$ is defined to be the set $P(A)=$ $\left\{\mathfrak{p} \in \operatorname{Spec}(A): P\right.$ holds for $\left.A_{\mathfrak{p}}\right\}$. We show that the $\left(S_{n}\right)$ locus of any $\left(S_{2}\right)$ local ring with Cohen-Macaulay formal fibres is an open $\operatorname{subset}$ of $\operatorname{Spec}(A)$ for all $n \geq 2$.

2.3. Corollary. If $A$ satisfies $\left(S_{2}\right)$ and all formal fibres of $A \longrightarrow \widehat{A}$ are CohenMacaulay, then for each $n \geq 0, S_{n}(A)$ is an open subset of $\operatorname{Spec}(A)$, in the Zariski topology. In particular, $C M(A)$ is an open subset of $\operatorname{Spec}(A)$.

Proof. It follows that $\widehat{A}$ is $\left(S_{2}\right)$. We assume that $n \geq 3$. Set $U_{i}=\operatorname{Spec}(A)-$ $\operatorname{Supp}_{A}\left(H^{i}\left(C_{A}(A)\right)\right), 1 \leq i \leq n-2$. Each $U_{i}$ is an open subset of $\operatorname{Spec} A$, because $\operatorname{Supp}_{A}\left(H^{i}\left(C_{A}(A)\right)\right)=V\left(0:_{A} H^{i}\left(C_{A}(A)\right)\right)$ by 2.2. Set $W=\cap_{i=1}^{n-2} U_{i}$. We show that $S_{n}(A)=W$. Let $\mathfrak{p} \in S_{n}(A)$; so that $A_{\mathfrak{p}}$ is $\left(S_{n}\right)$. Thus, by [SSc, Example 4.4], $H^{n}\left(C_{A_{\mathfrak{p}}}\left(A_{\mathfrak{p}}\right)\right)=0$ for $1 \leq i \leq n-2$. Therefore, by [S1, Theorem 3.5], we have that $\mathfrak{p} \in U_{n}$ for all $i, 1 \leq i \leq n-2$; that is $\mathfrak{p} \in W$. In a similar way, we have $W \subseteq S_{n}(A)$. $\square$

Next, we state a local duality property for the Cousin complexes of certain modules.

2.4. Theorem. (Local duality for certain Cousin complexes). Assume that all formal fibres of $A$ are Cohen-Macaulay, $M$ satisfies $\left(S_{2}\right)$, and that $\operatorname{Min}_{\widehat{A}}(\widehat{M})=$ 
$\operatorname{Assh}_{\widehat{A}}(\widehat{M})$. Then, for each $i \geq 0, D_{A} H^{i}\left(C_{A}(M)^{\prime}\right) \cong H_{\widehat{\mathfrak{m}}}^{s-i}\left(K_{\widehat{M}}\right)$, where $D_{A}:=$ $\operatorname{Hom}_{A}(-, E(A / \mathfrak{m}))$. Moreover, if $M$ admits a canonical module, then the completion signs on the right hand side of the above isomorphism can be removed.

Proof. Set $\bar{A}=A / 0:_{A} M$ and $\bar{A}=\widehat{A} / 0:_{\widehat{A}} \widehat{M}$. It is straightforward to see that $\overline{\widehat{A}}$ and $\widehat{\bar{A}}$ are isomorphic rings. Let $J^{\bullet}$ be the dualizing complex for $\widehat{A}$ and assume that $I^{\bullet}=\operatorname{Hom}_{\widehat{A}}\left(\overline{\hat{A}}, J^{\bullet}\right)$ such that $I^{0}=\operatorname{Hom}_{\widehat{A}}\left(\overline{\widehat{A}}, J^{d-s}\right)$. Hence $I^{\bullet}$ is the dualizing complex for $\widehat{\widehat{A}}$. As seen in the proof of $2.1, \widehat{M}$ satisfies $\left(S_{2}\right)$ as $\widehat{A}$-module. It is easy to see that $\widehat{M}$ also satisfies $\left(S_{2}\right)$ as $\overline{\widehat{A}}$-module. Since $\operatorname{dim}_{\bar{A}}(\widehat{M})=\operatorname{dim}(\widehat{\widehat{A}})$, we have, by the proof of [DT2, Lemma 3.1], the isomorphism of complexes

$$
C_{\overline{\widehat{A}}}(\widehat{M})^{\prime} \cong \operatorname{Hom}_{\overline{\hat{A}}}\left(K_{\widehat{M}}, I^{\bullet}\right) \text {. }
$$

Therefore, by 2.1 and [B-ZS, Corollary 2.5], we have

$$
D_{\overline{\widehat{A}}} H^{i}\left(C_{\overline{\widehat{A}}}(\widehat{M})^{\prime}\right) \cong H_{\widehat{\widehat{\mathfrak{m}}}}^{s-i}\left(K_{\widehat{M}}\right)
$$

for all $i \geq 0$.

On the other hand each formal fibre of $\bar{A}$ is also a formal fibre of $A$ and $\bar{A} \cong \widehat{\bar{A}}$. Hence, from 1.3, we have

$$
H^{i}\left(C_{\widehat{A}}(\widehat{M})^{\prime}\right) \cong H^{i}\left(C_{\bar{A}}(M)^{\prime}\right) \otimes_{\bar{A}} \widehat{\bar{A}}
$$

for all $i>0$. From (1) and (2), we obtain

$$
\operatorname{Hom}_{\widehat{\bar{A}}}\left(H^{i}\left(C_{\bar{A}}(M)^{\prime}\right) \otimes_{\bar{A}} \widehat{\bar{A}}, E_{\widehat{\widehat{A}}}(\widehat{\bar{A}} / \widehat{\overline{\mathfrak{m}}})\right) \cong H_{\widehat{\hat{\mathfrak{m}}}}^{s-i}\left(K_{\widehat{M}}\right) .
$$

The left hand side of (3) is isomorphic to

$$
\operatorname{Hom}_{\bar{A}}\left(H^{i}\left(C_{\bar{A}}(M)^{\prime}\right), \operatorname{Hom}_{\widehat{A}}\left(\widehat{\bar{A}}, E_{\widehat{A}}(\widehat{\bar{A}} / \widehat{\overline{\mathfrak{m}}})\right)\right)
$$

which, in turn, is isomorphic to $\operatorname{Hom}_{\bar{A}}\left(H^{i}\left(C_{\bar{A}}(M)^{\prime}\right), E_{\widehat{A}}(\widehat{\bar{A}} / \widehat{\overline{\mathfrak{m}}})\right)$. Thus, we have from (3), the isomorphism

$$
\operatorname{Hom}_{\bar{A}}\left(H^{i}\left(C_{\bar{A}}(M)^{\prime}\right), E_{\bar{A}}(\bar{A} / \overline{\mathfrak{m}})\right) \cong H_{\overline{\mathfrak{m}}}^{s-i}\left(K_{\widehat{M}}\right) .
$$

Assume that $N$ is an $A, \bar{A}$-bimodule such that, for $a \in A$ and $x \in N$, ax $=\bar{a} x$, where $-: A \longrightarrow \bar{A}$ is the natural map. Then we have

$$
\begin{aligned}
\operatorname{Hom}_{\bar{A}}\left(N, E_{\bar{A}}(\bar{A} / \overline{\mathfrak{m}})\right) & \cong \operatorname{Hom}_{\bar{A}}\left(N, \operatorname{Hom}_{A}(\bar{A}, E(A / \mathfrak{m}))\right) \\
& \cong \operatorname{Hom}_{A}\left(N \otimes_{\bar{A}} \bar{A}, E(A / \mathfrak{m})\right) \\
& \cong \operatorname{Hom}_{A}(N, E(A / \mathfrak{m})) .
\end{aligned}
$$


By Independence Theorem for the local cohomologies, we have $H_{\widehat{\mathfrak{m}}}^{s-i}\left(K_{\widehat{M}}\right) \cong$ $H_{\mathfrak{\mathrm { m }}}^{s-i}\left(K_{\widehat{M}}\right)$. Put all these together, we obtain, from (4) and 1.2, that

$$
\operatorname{Hom}_{A}\left(H^{i}\left(C_{A}(M)^{\prime}\right), E(A / \mathfrak{m})\right) \cong H_{\mathfrak{m}}^{s-i}\left(K_{\widehat{M}}\right), i=0,1, \ldots,
$$

as $A$ and $\widehat{A}$-modules.

If $M$ admits a canonical module $K_{M}$, we then have $\widehat{\left(K_{M}\right)} \cong K_{\widehat{M}}$, and by the Artinianness of $H_{\mathfrak{m}}^{s-i}\left(K_{M}\right)$, we get the final claim.

\section{Applichtions}

First we show that over a local ring with Cohen-Macaulay formal fibres, certain $f$-modules are also generalized Cohen-Macaulay modules. Recall that $M$ is called generalized Cohen-Macaulay (abbr. g.CM) if there exists $r \geq 1$ such that, for each system of parameters $x_{1}, \ldots, x_{s}$ for $M$ and for all $i=1, \ldots, s$,

$$
\mathfrak{m}^{r}\left[\left(\left(x_{1}, \ldots, x_{i-1}\right) M: x_{i}\right) /\left(x_{1}, \ldots, x_{i-1}\right) M\right]=0 .
$$

Note that, by [ScTC, (3.2) and (3.3)], $M$ is a g.CM module if and only if $H_{\mathfrak{m}}^{i}(M)$ is of finite length for all $i=0,1, \ldots, s-1$.

An $A$-module $M$ is called an $f$-module if for each system of parameters $x_{1}, \ldots, x_{s}$ for $M$

$$
\operatorname{Supp}_{A}\left[\left(\left(x_{1}, \ldots, x_{i-1}\right) M: x_{i}\right) /\left(x_{1}, \ldots, x_{i-1}\right) M\right] \subseteq\{\mathfrak{m}\}
$$

for all $i=1, \ldots, s$. It is clear that if $M$ is g.CM module then it is an $f$-module.

3.1. Theorem. (Compare [ScTC, (3.8)]). Assume that all formal fibres of A are Cohen-Macaulay. Let $M$ be an $A$-module such that $\operatorname{Min}_{\widehat{A}}(\widehat{M})=\operatorname{Assh}_{\widehat{A}}(\widehat{M})$. If $M$ is an $f$-module with $\operatorname{depth}_{A}(M) \geq 2$, then $M$ is a g.CM module.

Proof. By a straightforward argument and using the equivalent definition of $f-$ module [T, Lemma 1.2 (ii)], it can be shown that $M$ is $\left(S_{2}\right)$ and that $\operatorname{Min}_{A}(M)=$ $\operatorname{Assh}_{A}(M)$.

Now, by [T, Lemma 1.2 (iv)], the $M$-height filtration of $\operatorname{Spec}(A)$ is the same as the $M$-dimension filtration $\mathcal{D}$ of $\operatorname{Spec}(A)$, where $\mathcal{D}=\left(D_{i}\right)_{i \geq 0}, D_{i}=\left\{\mathfrak{p} \in \operatorname{Supp}_{A}(M)\right.$ : $\operatorname{dim}(A / \mathfrak{p}) \leq s-i\}$. Thus, by [DT1, Lemma 3.1], there exists an isomorphism

$$
C_{A}(M)=C(\mathcal{D}, M) \cong C(\mathcal{U}, M)
$$

(over $\operatorname{Id}_{M}$ ), where $C(\mathcal{U}, M)$ is the complex of modules of generalized fractions on $M$ with respect to the chain of triangular subsets $\mathcal{U}=\left(U_{i}\right)_{i \geq 1}$ on $A$, defined by

$$
\begin{gathered}
U_{i}=\left\{\left(x_{1}, \cdots, x_{i}\right) \in A^{i}: \text { there exists } j \text { with } 0 \leq j \leq i \text { such that } x_{1}, \cdots, x_{j}\right. \\
\text { is an s.s.o.p. for } \left.M \text { and } x_{j+1}=\cdots=x_{i}=1\right\}
\end{gathered}
$$

(See [DT1] for details). By [SZ, Corollary 2.3 and Theorem 2.4], 


$$
H^{i-1}\left(C_{A}(M)\right) \cong H_{\mathfrak{m}}^{i}(M), i=1, \cdots, s-1 .
$$

Therefore, by Theorem 2.1, $H_{\mathfrak{m}}^{i}(M)$ is of finite length for all $i=0,1, \cdots, s-1$. $\square$

3.2. Corollary. Assume that all formal fibres of $A$ are Cohen-Macaulay. If $A$ is an f-ring with $\operatorname{depth}(A) \geq 2$, then $A$ is a g.CM ring.

Proof. As we have seen in the proof of 3.1, $A$ is $\left(S_{2}\right)$. By [M, Theorem 23.9], $\widehat{A}$ satisfies $\left(S_{2}\right)$. Thus $\operatorname{Min}(\widehat{A})=\operatorname{Assh}(\widehat{A})$ (see $[\mathrm{AG}$, Lemma 1.1]). Now the result follows from Theorem 3.1.

Our next application studies the injectivity of the terms of the Cousin complex $C_{A}(M)$.

In [S2], a finitely generated $A$-module $M$ is defined to be a Gorenstein $A$-module whenever its Cousin complex provides a minimal injective resolution. It is also proved that if $A$ admits a canonical module $\Omega$, then any Gorenstein $A$-module is isomorphic to the direct sum of a finite number of copies of $\Omega$ [S3, Theorem 2.1].

It is known that if $A$ does not have a canonical module and has a Gorenstein module, then it has a unique indecomposable Gorenstein module $G$ and every Gorenstein $A$-module is isomorphic to a direct sum of a finite number of copies $G$ (see[FFGR and S2]). Here we extend this result and show that for any finitely generated module $M$, over a complete $\left(S_{2}\right)$ local ring $A$ which satisfies $\left(S_{2}\right)$, if $0:{ }_{A} M=0$ and $C_{A}(M)^{\prime}$ is an injective complex, then $M$ is isomorphic to a direct sum of copies of a uniquely determined indecomposable one.

3.3. Theorem. Let A satisfy $\left(S_{2}\right)$ and suppose that it possesses a dualizing complex. Assume that $M$ satisfies $\left(S_{2}\right)$ and $0: A=0$. The following statements are equivalent:

(i) $C_{A}(M)^{\prime}$ is an injective complex;

(ii) $M$ is isomorphic to a direct sum of a finite number of copies of the canonical module $K$ of the ring $A$.

Proof. (i) $\Rightarrow$ (ii). We do not need $A$ to satisfy $\left(S_{2}\right)$ in this part. The proof is a straightforward adaptation of the argument in [S3, Theorem 2.1(v)]. Let $K$ denote the canonical module of $A$.

Let

$$
I^{\bullet}: 0 \longrightarrow I^{0} \longrightarrow I^{1} \longrightarrow \cdots \longrightarrow I^{d} \longrightarrow 0
$$


be the dualizing complex for $A$ so that $K=H^{0}\left(I^{\bullet}\right)$. By the proof of [DT2, Lemma 3.1], $C_{A}(M) \cong \operatorname{Hom}_{A}\left(K_{M}, I^{\bullet}\right)^{*}$, where $K_{M}=\operatorname{Hom}_{A}(M, K)$. Hence all cohomology modules of $C_{A}(M)$ are finitely generated (see [S4, Lemma 3.4(ii)]). By [S5, Theorem], $\operatorname{Hom}_{A}\left(K_{M}, I^{d}\right) \cong H_{\mathfrak{m}}^{d}(M)$. As $H_{\mathfrak{m}}^{d}(M)$ is an Artinian injective $A$-module, we may write $H_{\mathfrak{m}}^{d}(M) \cong \oplus_{i=1}^{n} E(A / \mathfrak{m})$, say. Using the Matlis functor $\operatorname{Hom}_{A}(-, E(A / \mathfrak{m}))$ and that $I^{d}=E(A / \mathfrak{m})$, we obtain $K_{M} \otimes_{A} \widehat{A} \cong\left(\oplus_{i=1}^{n} A\right) \otimes_{A} \widehat{A}$. This implies, by[HK, Lemma 5.8], that $K_{M} \cong \oplus_{i=1}^{n} A$. Hence we have $H_{\mathfrak{m}}^{d}\left(K_{M}\right) \cong \oplus_{i=1}^{n} H_{\mathfrak{m}}^{d}(A)$.

On the other hand, by Grothendieck's Local Duality Theorem [B-ZS, Corollary 2.5] and the fact that $M$ satisfies $\left(S_{2}\right)$ so $C_{A}(M)$ is exact at point $-1,0$ (see [SSc, Example 4.4]), we obtain

$$
H_{\mathfrak{m}}^{d}\left(K_{M}\right) \cong \operatorname{Hom}_{A}\left(H^{0}\left(C_{A}(M)^{\prime}\right), E(A / \mathfrak{m})\right) \cong \operatorname{Hom}_{A}(M, E(A / \mathfrak{m})) .
$$

By applying the Matlis functor again, we get $M \otimes_{A} \widehat{A} \cong \operatorname{Hom}_{A}\left(H_{\mathfrak{m}}^{d}\left(K_{M}\right), E(A / \mathfrak{m})\right) \cong$ $\operatorname{Hom}_{A}\left(\oplus_{i=1}^{n} H_{\mathfrak{m}}^{d}(A), E(A / \mathfrak{m})\right) \cong\left(\oplus_{i=1}^{n} K\right) \otimes_{A} \widehat{A}$. Now, by [HK, Lemma 5.8], $M \cong$ $\oplus_{i=1}^{n} K$.

(ii) $\Rightarrow\left(\right.$ i). We have $\operatorname{Supp}_{A}(M)=\operatorname{Supp}_{A}(K)=\operatorname{Spec}(A)$ (see $[\mathrm{A},(1.8)]$ and $[\mathrm{AG}$, Lemma 1.1]). It is routine to check that $C_{A}(M) \cong \oplus_{i=1}^{n} C_{A}(K)$. As $\operatorname{Min} A=\operatorname{Assh} A$ and the dimension filtration and the height filtration of $\operatorname{Spec}(A)$ are the same (see $[\mathrm{A},(1.9)])$, the claim follows by [DT1, Corollary 3.4]. $\square$

3.4. Corollary. Assume that $\widehat{A}$ satisfies $\left(S_{2}\right)$. Then the following statements are equivalent :

(i) $C_{\widehat{A}}(\widehat{A})^{\prime}$ is an injective complex of $\widehat{A}$-modules;

(ii) $A$ is the canonical module of $A$.

Moreover, if $A$ satisfies one of the above equivalent conditions, then $A$ is Gorenstein if and only if $\widehat{A}$ satisfies $\left(S_{n}\right)$ for some $n \geq(1 / 2) \operatorname{dim} A+1$.

Proof. (i) $\Rightarrow$ (ii). Set $\Omega$ for the canonical module of $\widehat{A}$. By $3.3, \widehat{A} \cong \Omega^{n}$ for some $n$. Thus $H_{\widehat{\mathfrak{m}}}^{d}(\widehat{A}) \cong \oplus_{i=1}^{n} H_{\mathfrak{\mathfrak { m }}}^{d}(\Omega) \cong \oplus_{i=1}^{n} E(\widehat{A} / \widehat{\mathfrak{m}})$ and, by applying $\operatorname{Hom}_{\widehat{A}}(-, E(\widehat{A} / \widehat{\mathfrak{m}}))$, we get $\Omega \cong \widehat{A}^{n}$. Thus $\widehat{A}^{n^{2}}=\widehat{A}$, which implies $n=1$ and so $A$ is the canonical module of $A$.

(ii) $\Rightarrow$ (i). As $\widehat{A}$ is the canonical module of $\widehat{A}$ and $\widehat{A}$ satisfies $\left(S_{2}\right), C_{\widehat{A}}(\widehat{A})^{\prime}$ is the dualizing complex of $\widehat{A}$ [DT1, Corollary 3.4].

For the last part, we may assume that $A$ is complete. By [SSc, Example 4.4], $C_{A}(A)$ is exact at points $-1,0,1, \ldots, n-2$, from which it follows, by Theorem 2.4, that $H_{\mathfrak{m}}^{\operatorname{dim} A-i}(A)=0$ for $0<i \leq n-2$. On the other hand, as $A$ satisfies $\left(S_{n}\right)$, $H_{\mathfrak{m}}^{i}(A)=0$ for all $i<\min \{d, n\}$. As $\operatorname{dim} A-(n-2) \leq n$, it follows that $H_{\mathfrak{m}}^{i}(A)=0$ 
for all $i<d$, which imply the exactness of $C_{A}(A)$. The other side is trivial. $\square$

\section{Canonical modules of NON-LOCAL Rings}

Recall that, for a Noetherian (not necessarily local) ring $R$, the canonical module of $\mathrm{R}$ (if it exists) is a finite $R$ - module $K$ such that $K_{\mathfrak{m}}$, the localization of $K$ at any maximal ideal $\mathfrak{m}$ of $R$, is the canonical module of $R_{\mathfrak{m}}$. In order to generalize our results to the non-local case one might ask whether a canonical module exists even when $R$ possesses a dualizing complex. We will show that, if $R$ satisfies $\left(S_{2}\right)$ and all formal fibres of $R_{\mathfrak{m}}$, for any maximal ideal $\mathfrak{m}$ of $R$, are Cohen-Macaulay, then existence of a canonical module for $R$ is equivalent to the statement that $R$ possesses a dualizing complex.

Throughout, $R$ is a Noetherian ring of finite dimension which is not necessarily local.

Assume that $R$ possesses a dualizing complex $I^{\bullet}$ and $t\left(\mathfrak{p} ; I^{\bullet}\right), \mathfrak{p} \in \operatorname{Spec} R$, denotes the unique integer $i$ for which $\mathfrak{p}$ occurs in $I^{i}$ (see [H, page 23]).

4.1. Proposition. Assume that $R$ satisfies $\left(S_{2}\right)$ and that it possesses a dualizing complex $I^{\bullet}$. If $\mathfrak{p}, \mathfrak{q} \in \operatorname{Min}(R)$ such that $\mathfrak{p} \subseteq \mathfrak{r}$ and $\mathfrak{q} \subseteq \mathfrak{r}$ for some $\mathfrak{r} \in \operatorname{Spec}(R)$, then $t\left(\mathfrak{p} ; I^{\bullet}\right)=t\left(\mathfrak{q} ; I^{\bullet}\right)$.

Proof. We may assume that $R$ is a local ring and that its maximal ideal is $\mathfrak{r}$. As $R$ satisfies $\left(S_{2}\right)$ and possesses a dualizing complex, then $\operatorname{Min}(R)=\operatorname{Assh}(R)[\mathrm{A} ; 1.1]$. Therefore $t\left(\mathfrak{p} ; I^{\bullet}\right)=t\left(\mathfrak{q} ; I^{\bullet}\right)$.

4.2. Notation. Assume that $R$ satisfies $\left(S_{2}\right)$ and that

$$
I^{\bullet}: 0 \longrightarrow I^{0} \stackrel{\delta^{0}}{\longrightarrow} I^{1} \stackrel{\delta^{1}}{\longrightarrow} \cdots \stackrel{\delta^{l-1}}{\longrightarrow} I^{l} \longrightarrow 0
$$

is a dualizing complex for $R$. It follows that $\operatorname{Ass}_{R}\left(I^{0}\right) \subseteq \operatorname{Min} R$. Assume that $\operatorname{Ass}_{R}\left(I^{0}\right) \neq \operatorname{Min}(R)$. Let $r$ be the greatest integer such that $X:=\operatorname{Min}(R) \bigcap \operatorname{Ass}_{R}\left(I^{r}\right) \neq$ $\emptyset$. Set, for each $i \geq 0$,

$$
\begin{gathered}
X_{i}=\left\{\mathfrak{p} \in \operatorname{Ass}_{R}\left(I^{i}\right): \mathfrak{p} \text { contains some element of } X\right\} ; \\
X_{i}^{\prime}=\operatorname{Ass}_{R}\left(I^{i}\right) \backslash X_{i} ; \\
I_{1}^{i}=\oplus_{\mathfrak{p} \in X_{i}} E(A / \mathfrak{p}), I_{2}^{i}=\oplus_{\mathfrak{p} \in X_{i}^{\prime}} E(A / \mathfrak{p}),
\end{gathered}
$$

so that we have $I^{i}=I_{1}^{i} \oplus I_{2}^{i}$. 
4.3. Proposition. With the notations as in 4.2,

$$
\operatorname{Hom}_{R}\left(I_{1}^{i}, I_{2}^{i+1}\right)=0=\operatorname{Hom}_{R}\left(I_{2}^{i}, I_{1}^{i+1}\right) .
$$

Proof. If $\operatorname{Hom}_{R}\left(I_{1}^{i}, I_{2}^{i+1}\right) \neq 0$, then $\operatorname{Hom}_{R}\left(I_{1}^{i}, E(R / \mathfrak{p})\right) \neq 0$ for some $\mathfrak{p} \in X_{i+1}^{\prime}$. Assume that $f: I_{1}^{i} \longrightarrow E(R / \mathfrak{p})$ is an $R$-homomorphism and that $x \in I_{1}^{i}$. Let $x=x_{1}+\cdots+x_{s}$, where $x_{j} \in E\left(R / \mathfrak{p}_{j}\right), 1 \leq j \leq s$. By definition of $X_{i+1}^{\prime}$, we have $\mathfrak{p}_{1} \bigcap \cdots \bigcap \mathfrak{p}_{s} \nsubseteq \mathfrak{p}$. Take $t \in \mathfrak{p}_{1} \bigcap \cdots \bigcap \mathfrak{p}_{s} \backslash \mathfrak{p}$. Hence $t^{m} x=0$ for some positive integer $m$. On the other hand the map $E(R / \mathfrak{p}) \stackrel{t^{m}}{\longrightarrow} E(R / \mathfrak{p})$ is an isomorphism. Thus $t^{m} f(x)=f\left(t^{m} x\right)=0$ implies that $f(x)=0$.

To show that $\operatorname{Hom}_{R}\left(I_{2}^{i}, I_{1}^{i+1}\right)=0$, we may assume, on the contrary, that $\operatorname{Hom}_{R}\left(I_{2}^{i}, E(R / \mathfrak{p})\right) \neq 0$ for some $\mathfrak{p} \in X_{i+1}$. So we may assume that $\mathfrak{p}^{\prime} \subseteq \mathfrak{p}$, for some $\mathfrak{p}^{\prime} \in X_{i}^{\prime}$. By localizing at $\mathfrak{p}$, we get $\operatorname{Min}\left(R_{\mathfrak{p}}\right)=\operatorname{Assh}\left(R_{\mathfrak{p}}\right)$, because $R_{\mathfrak{p}}$ satisfies $\left(S_{2}\right)$ and $R_{\mathfrak{p}}$ possesses a dualizing complex. As $\mathfrak{p}^{\prime} R_{\mathfrak{p}}$ contains a minimal element $\mathfrak{q} R_{\mathfrak{p}} \in \operatorname{Assh}\left(R_{\mathfrak{p}}\right)$, we get, by $4.1, t\left(\mathfrak{q} ; I^{\bullet}\right)=r$ and that $\mathfrak{q} \in X$. This contradicts with the definition of $X_{i}^{\prime}$.

4.4. Theorem. Assume that $R$ satisfies $\left(S_{2}\right)$ and that it possesses a dualizing complex. Then $R$ possesses a dualizing complex

$$
J^{\bullet}: 0 \longrightarrow J^{0} \longrightarrow J^{1} \longrightarrow \cdots \longrightarrow J^{d} \longrightarrow 0, d=\operatorname{dim} R
$$

such that $\operatorname{Ass}_{R}\left(J^{0}\right)=\operatorname{Min}(R)$. In particular $R$ admits a canonical module.

Proof. The proof is influenced by [H, Lemma 3.1]. Suppose that

$$
I^{\bullet}: 0 \longrightarrow I^{0} \stackrel{\delta^{0}}{\longrightarrow} I^{1} \stackrel{\delta^{1}}{\longrightarrow} \cdots \stackrel{\delta^{l-1}}{\longrightarrow} I^{l} \longrightarrow 0
$$

is the dualizing complex for $R$. Assume further that $\operatorname{Ass}\left(I^{0}\right) \neq \operatorname{Min}(R)$ and that $r$ is the greatest integer with $X:=\operatorname{Min}(R) \bigcap \operatorname{Ass}_{R}\left(I^{r}\right) \neq \emptyset$. We set $X_{i}, X_{i}^{\prime}, I_{1}^{i}$ and $I_{2}^{i}$ as in 4.2. Note that $I_{1}^{i}=0$ and $I_{2}^{i}=I^{i}$ for $0 \leq i<r$ (see [S6, Lemma 3.3]).

We construct a dualizing complex

$$
J^{\bullet}: 0 \longrightarrow J^{0} \stackrel{\eta_{0}}{\longrightarrow} J^{1} \stackrel{\eta_{1}}{\longrightarrow} \cdots
$$

as follows. Set $J^{i}=I_{1}^{i+r} \oplus I_{2}^{i}$ for all $i \geq 0$, and define $\eta^{i}: J^{i} \longrightarrow J^{i+1}$ by $\eta^{i}(x+y)=\delta_{1}^{i+r}(x)+\delta_{2}^{i}(y)$ for $x \in I_{1}^{i+r}, y \in I_{2}^{i}$, where $\delta_{1}^{j}:=\left.\delta^{j}\right|_{I_{1}^{j}}$ and $\delta_{2}^{j}:=\left.\delta^{j}\right|_{I_{2}^{j}}, j \geq 0$. It follows from Proposition 4.3 that $J^{\bullet}$ is a complex. To show that $H^{i}\left(J^{\bullet}\right)$ is a finitely generated $R$-module for all $i \geq 0$, we note that, by a straightforward argument, there are two natural isomorphisms

$$
\begin{gathered}
H^{i}\left(I^{\bullet}\right) \cong\left(\operatorname{Ker} \delta_{1}^{i} / \operatorname{Im} \delta_{1}^{i-1}\right) \oplus\left(\operatorname{Ker} \delta_{2}^{i} / \operatorname{Im} \delta_{2}^{i-1}\right) \\
H^{i}\left(J^{\bullet}\right) \cong\left(\operatorname{Ker} \delta_{1}^{i+r} / \operatorname{Im} \delta_{1}^{i+r-1}\right) \oplus\left(\operatorname{Ker} \delta_{2}^{i} / \operatorname{Im} \delta_{2}^{i-1}\right), i \geq 0
\end{gathered}
$$


Therefore $J^{\bullet}$ is a dualizing complex for $A$. Now we have $J^{0}=I_{1}^{r} \oplus I^{0}$ and thus $\operatorname{Ass}_{R}\left(I^{0}\right) \varsubsetneqq \operatorname{Ass}_{R}\left(J^{0}\right)$. So after a finite number of steps we are finished.

Finally, let $J^{\bullet}$ be a dualizing complex with $\operatorname{Min}(R)=\operatorname{Ass}_{R}\left(J^{0}\right)$. For each $\mathfrak{m} \in$ $\operatorname{Max}(R)$, the complex

$$
\left.0 \longrightarrow\left(J^{0}\right)_{\mathfrak{m}} \longrightarrow\left(J^{1}\right)_{\mathfrak{m}} \longrightarrow \cdots \longrightarrow\left(J^{t\left(\mathfrak{m} ; J^{\bullet}\right.}\right)\right)_{\mathfrak{m}} \longrightarrow 0
$$

is the dualizing complex for $R_{\mathfrak{m}}$, so that, by Grothendieck's Local Duality Theorem [B-ZS, Corollary 2.5], $\left(H^{0}\left(J^{\bullet}\right)\right)_{\mathfrak{m}}$ is the canonical module of $R_{\mathfrak{m}}$. Thus $H^{0}\left(J^{\bullet}\right)$ is a canonical module of $R$.

As an application, we can give a partial generalization of [BH, Proposition 3.3.18].

4.5. Theorem. Assume that $R$ satisfies $\left(S_{2}\right)$ and possesses a dualizing complex

$$
I^{\bullet}: 0 \longrightarrow I^{0} \longrightarrow I^{1} \longrightarrow \cdots \longrightarrow I^{d} \longrightarrow 0
$$

with $d=\operatorname{dim} R, I^{i}=\underset{\mathrm{htp}=i}{\oplus} E(R / \mathfrak{p}), i=0,1, \cdots$ and that $K_{R}$ is a canonical module of $R$.

(a) The following conditions are equivalent:

(i) $K_{R}$ has a rank;

(ii) $\operatorname{rank} K_{R}=1$;

(iii) $R$ is generically Gorenstein (that is $R_{\mathfrak{p}}$ is a Gorenstein ring for all minimal prime ideals $\mathfrak{p}$ of $R$ ).

(b) If $K_{R}$ satisfies $\left(S_{3}\right)$ and the equivalent conditions of $(a)$ hold, then $K_{R}$ can be identified with an ideal of height 1 or equals $R$. In the first case $R / K_{R}$, is an $\left(S_{2}\right)$ ring with the canonical module $R / K_{R}$, the ring itself.

Proof. The proof is parallel to that of [BH, Proposition 3.3.18] and we present it for the convenience of the reader.

(a). (i) $\Rightarrow$ (ii) $\Rightarrow$ (iii). Set $Q$ to be the ring of total fractions of $R$ and let $K_{R} \otimes_{R} Q$ be a free $Q$-module of rank $r$, say. Let $\mathfrak{p} \in \operatorname{Min}(R)$. As $\operatorname{Min}(R)=\operatorname{Ass}(R)=\operatorname{Ass}_{R}\left(K_{R}\right)$ (see [DT1, 1.3]), we know that $K_{R_{\mathfrak{p}}} \cong\left(K_{R}\right)_{\mathfrak{p}}$ and, by [BH, Proposition 1.4.3], the $R_{\mathfrak{p}}$-module $\left(K_{R}\right)_{\mathfrak{p}}$ is free of rank $r$. As the dualizing complex of $R_{\mathfrak{p}}$ is $\left(I^{\bullet}\right)_{\mathfrak{p}}: 0 \longrightarrow$ $I_{\mathfrak{p}}^{0} \longrightarrow 0$, we get $\left(R_{\mathfrak{p}}\right)^{r} \cong\left(K_{R}\right)_{\mathfrak{p}} \cong E(R / \mathfrak{p})$ from which it follows that $r=1$, and thus $R_{\mathfrak{p}}$ is Gorenstein.

(iii) $\Rightarrow($ i). Note that $\operatorname{Min}(R)=\operatorname{Ass}(R)$, and thus [BH, Proposition 1.4.3] implies that $K_{R}$ has rank 1 .

As $\operatorname{Ass} R=\operatorname{Ass}\left(K_{R}\right), K$ is torsion free. Thus [BH, 1.4.18] implies that $K_{R}$ is isomorphic to a sub-module of a free $R$-module of rank 1 , and it may be identified with an ideal of $R$ which we again denote by $K_{R}$. 
If $\operatorname{dim} R=0$, we get $K_{R} \cong R$, so we may assume $\operatorname{dim} R>0$, and also $K_{R}$ is a proper ideal of $R$. By [BH, Proposition 1.4.3], $K_{R}$ has a free sub-module $\mathfrak{a}$, which is also an ideal of $R$ of rank 1 . Assuming $\mathfrak{a}=x R$ with $x$ is a base for $\mathfrak{a}, x$ is $R$-regular and $K_{R}$-regular. Let $\mathfrak{p}$ be a prime ideal containing $K_{R}$. Applying the functor $\operatorname{Hom}_{R_{\mathfrak{p}}}\left(-,\left(I^{\bullet}\right)_{\mathfrak{p}}\right)$ on the exact sequence $0 \longrightarrow K_{R} R_{\mathfrak{p}} \longrightarrow R_{\mathfrak{p}} \longrightarrow R_{\mathfrak{p}} / K_{R} R_{\mathfrak{p}} \longrightarrow 0$, we get the exact sequence

$$
\begin{aligned}
0 & \longrightarrow H^{0}\left(\operatorname{Hom}_{R_{\mathfrak{p}}}\left(R_{\mathfrak{p}} / K_{R} R_{\mathfrak{p}},\left(I^{\bullet}\right)_{\mathfrak{p}}\right)\right) \longrightarrow H^{0}\left(\left(I^{\bullet}\right)_{\mathfrak{p}}\right) \longrightarrow H^{0}\left(\operatorname{Hom}_{R_{\mathfrak{p}}}\left(K_{R} R_{\mathfrak{p}},\left(I^{\bullet}\right)_{\mathfrak{p}}\right)\right) \\
& \longrightarrow H^{1}\left(\operatorname{Hom}_{R_{\mathfrak{p}}}\left(R_{\mathfrak{p}} / K_{R} R_{\mathfrak{p}},\left(I^{\bullet}\right)_{\mathfrak{p}}\right)\right) \longrightarrow H^{1}\left(\left(I^{\bullet}\right)_{\mathfrak{p}}\right) \longrightarrow \cdots
\end{aligned}
$$

Note that $H^{1}\left(I^{\bullet}\right)_{\mathfrak{p}}=0$ as $K_{R_{\mathfrak{p}}}$ satisfies $\left(S_{3}\right)$ (see [DT2, Proposition 2.5]).

On the other hand, we have $H^{0}\left(\operatorname{Hom}_{R_{\mathfrak{p}}}\left(R_{\mathfrak{p}} / K_{R} R_{\mathfrak{p}},\left(I^{\bullet}\right)_{\mathfrak{p}}\right)\right) \cong 0:_{K_{R} R_{\mathfrak{p}}} K_{R} R_{\mathfrak{p}} \subseteq$ $0:_{R_{\mathfrak{p}}} K_{R} R_{\mathfrak{p}}=0$ because $K_{R} R_{\mathfrak{p}}$ is the canonical module of $R_{\mathfrak{p}}$ and $R_{\mathfrak{p}}$ satisfies $\left(S_{2}\right)$. Therefore we get the exact sequence

$$
o \longrightarrow K_{R} R_{\mathfrak{p}} \longrightarrow R_{\mathfrak{p}} \longrightarrow H^{1}\left(\operatorname{Hom}_{R_{\mathfrak{p}}}\left(R_{\mathfrak{p}} / K_{R} R_{\mathfrak{p}},\left(I^{\bullet}\right)_{\mathfrak{p}}\right)\right) \longrightarrow 0
$$

which implies that $H^{1}\left(\operatorname{Hom}_{R_{\mathfrak{p}}}\left(R_{\mathfrak{p}} / K_{R} R_{\mathfrak{p}},\left(I^{\bullet}\right)_{\mathfrak{p}}\right)\right) \cong\left(R / K_{R}\right)_{\mathfrak{p}}$. It follows that $\operatorname{dim}\left(R_{\mathfrak{p}} / K_{R} R_{\mathfrak{p}}\right)=$ htp -1 . Using the Grothendieck local duality shows that $R_{\mathfrak{p}} / K_{R} R_{\mathfrak{p}}$ is the canonical module of $R_{\mathfrak{p}} / K_{R} R_{\mathfrak{p}}$. As $K_{R} R_{\mathfrak{p}}$ contains an $R_{\mathfrak{p}}$-regular element, we have $\operatorname{ht}_{R_{\mathfrak{p}}}\left(K_{R} R_{\mathfrak{p}}\right) \geq 1$. Since $\operatorname{dim}\left(R_{\mathfrak{p}} / K_{R} R_{\mathfrak{p}}\right)=$ htp -1 , we get $\operatorname{ht}\left(K_{R}\right)=1$.

For the final part, we may assume that $\operatorname{dim} R>3$. As $R$ is $\left(S_{2}\right)$ and $K_{R}$ is $\left(S_{3}\right)$, from the exact sequence $H_{\mathfrak{p} R_{\mathfrak{p}}}^{i}\left(R_{\mathfrak{p}}\right) \longrightarrow H_{\mathfrak{p} R_{\mathfrak{p}}}^{i}\left(R_{\mathfrak{p}} / K_{R} R_{\mathfrak{p}}\right) \longrightarrow H_{\mathfrak{p} R_{\mathfrak{p}}}^{i+1}\left(K_{R} R_{\mathfrak{p}}\right)$, we get $H_{\mathfrak{p} R_{\mathfrak{p}}}^{i}\left(R_{\mathfrak{p}} / K_{R} R_{\mathfrak{p}}\right)=0$ for $i=0,1$. This shows that $R_{\mathfrak{p}} / K_{R} R_{\mathfrak{p}}$ satisfies $\left(S_{2}\right)$.

We can also generalize[DT1, Corollary 3.4].

4.6. Theorem. Assume that $R$ satisfies $\left(S_{2}\right)$, and that $\operatorname{dim} R<\infty$. The following statements are equivalent.

(i) $R$ possesses a dualizing complex;

(ii) $R$ admits a canonical module $K$, and $C(\mathcal{H}, K)^{\prime}$, the induced complex of the Cousin complex of $K$ with respect to the height filtration $\mathcal{H}=\left(H_{i}\right)_{i \geq 0}$ with $H_{i}=\{\mathfrak{p} \in \operatorname{Spec}(R): \operatorname{ht}(\mathfrak{p}) \geq i\}$, is a dualizing complex for $R$;

(iii) $R$ admits a canonical module $K$ and $H^{i}\left(C(\mathcal{H}, K)^{\prime}\right)$ is finitely a generated $R$-module for all $i \geq 1$.

Proof. (i) $\Rightarrow($ ii). By 4.4, there exists a dualizing complex

$$
I^{\bullet}: 0 \longrightarrow I^{0} \stackrel{\delta^{0}}{\longrightarrow} I^{1} \stackrel{\delta^{1}}{\longrightarrow} \cdots
$$


for $R$ such that $\operatorname{Ass}_{R}\left(I^{0}\right)=\operatorname{Min}(R)$. Set $K=\operatorname{Ker} \delta^{0}$. As seen in 4.4, $K$ is a canonical module of $R$ and $\operatorname{Ass}_{R}(K)=\operatorname{Min}(R)$. Now, by [DT1, Theorem 2.4(iv)], $C(\mathcal{H}, K)^{\prime}$ is a dualizing complex for $R$.

(ii) $\Rightarrow$ (iii) is clear.

(iii) $\Rightarrow(\mathrm{i})$. For each $\mathfrak{m} \in \operatorname{Max}(R)$, we have, by [S1, Theorem 3.5], $C(\mathcal{H}, K)_{\mathfrak{m}} \cong$ $C\left(\mathcal{H}_{\mathfrak{m}}, K_{\mathfrak{m}}\right)$, where $\mathcal{H}_{\mathfrak{m}}$ is the height filtration of $R_{\mathfrak{m}}$. Therefore, by [DT1, Corollary 3.4], $\left(C(\mathcal{H}, K)^{\prime}\right)_{\mathfrak{m}}$ is a dualizing complex for $R_{\mathfrak{m}}$. Since $R_{\mathfrak{p}}$ satisfies $\left(S_{2}\right)$ for all $\mathfrak{p} \in \operatorname{Spec}(R)$, by the same argument as in the proof of [DT1, Corollary 3.4], each term of $C(\mathcal{H}, K)^{\prime}$ is an injective module. Thus, by [S4, Theorem 4.2], $C(\mathcal{H}, K)^{\prime}$ is a dualizing complex for $R$.

\section{INDECOMPOSABLE INJECTIVE MODULES STRUCTURE}

In this section, by using of a particular dualizing complex for an $\left(S_{2}\right)$ Noetherian ring $R$ of finite dimension, we give an explicit description for the structure of all indecomposable injective modules. In [DT1, Corollary 3.3], it is shown that for each $\mathfrak{p} \in \operatorname{Spec}(R)$, there exists a finitely generated $R$-module $T$, depending on $\mathfrak{p}$, such that $E(R / \mathfrak{p})$ is a module of generalized fractions of $T$. Here we will show that $T$ can be replaced by a canonical module of $R$ and that it does not depend on $\mathfrak{p}$.

Our approach involves the concept of a chain of triangular subsets on $R$ explained in $\left[\mathrm{O}\right.$, page 420]. Such a chain $\mathcal{U}=\left(U_{i}\right)_{i \geq 1}$ determines a complex $C(\mathcal{U}, M)$ of modules of generalized fractions on an $R$-module $M$, that is

$$
C(\mathcal{U}, M): 0 \longrightarrow M \stackrel{e^{0}}{\longrightarrow} U_{1}^{-1} M \stackrel{e^{1}}{\longrightarrow} \cdots \stackrel{e^{i-1}}{\longrightarrow} U_{i}^{-i} M \stackrel{e^{i}}{\longrightarrow} U_{i+1}^{-i-1} M \stackrel{e^{i+1}}{\longrightarrow} \cdots
$$

in which $e^{0}(m)=\frac{m}{(1)}$ for all $m \in M$ and $e^{i}\left(\frac{m}{\left(u_{1}, \cdots, u_{i}\right)}\right)=\frac{m}{\left(u_{1}, \cdots, u_{i}, 1\right)}$ for all $i \geq 1$, $m \in M$, and $\left(u_{1}, \cdots, u_{i}\right) \in U_{i}$. Note that in the complex $C(\mathcal{U}, M), U_{i+1}^{-i-1} M$ is regarded as the $i$-th term, so that $H^{i}(C(\mathcal{U}, M))=\operatorname{Ker} e^{i+1} / \operatorname{Im} e^{i}, i \geq 0$,

and $H^{-1}(C(\mathcal{U}, M))=\operatorname{Ker} e^{0}$.

Assume that $R$ satisfies $\left(S_{2}\right)$ and possesses a dualizing complex, so that $R$ possesses a dualizing complex

$$
I^{\bullet}: 0 \longrightarrow I^{0} \stackrel{\delta^{0}}{\longrightarrow} I^{1} \stackrel{\delta^{1}}{\longrightarrow} \cdots \stackrel{\delta^{d-1}}{\longrightarrow} I^{d} \longrightarrow 0, d=\operatorname{dim} R,
$$

such that $\operatorname{Ass}_{R}\left(I^{0}\right)=\operatorname{Min}(R)$. Set $K=\operatorname{Ker} \delta^{0}$, and consider the induced extended complex

$$
I^{*}: 0 \longrightarrow K \hookrightarrow I^{0} \stackrel{\delta^{0}}{\longrightarrow} I^{1} \stackrel{\delta^{1}}{\longrightarrow} \cdots \longrightarrow I^{d} \longrightarrow 0, .
$$

For each $\mathfrak{p} \in \operatorname{Ass}_{R}\left(I^{0}\right)$, the complex $0 \longrightarrow\left(I^{0}\right)_{\mathfrak{p}} \longrightarrow 0$ is the dualizing complex for $R_{\mathfrak{p}}$, so that $K_{\mathfrak{p}} \cong E(R / \mathfrak{p})$. Hence $\operatorname{Ass}_{R}(K)=\operatorname{Min}(R)$. Thus, by [DT1, Proposition 
$3.2]$, there is a unique isomorphism of complexes (over $\operatorname{Id}_{K}$ ) from $I^{*}$ to $C(\mathcal{V}, K)$, the complex of modules of generalized fractions on $K$ with respect to the chain of triangular subsets $\mathcal{V}=\left(V_{i}\right)_{i \geq 1}$ on $R$, defined by

$$
V_{i}=\left\{\left(v_{1}, \cdots, v_{i}\right) \in R^{i}: \operatorname{ht}_{R}\left(\left(v_{1}, \cdots, v_{j}\right)\right) \geq j \text { for all } j \text { with } 1 \leq j \leq i\right\} .
$$

Now, we restate [DT1, Corollary 3.3] in a more appropriate form.

5.1. Corollary. Assume that $R$ satisfies $\left(S_{2}\right)$ and that it possesses a dualizing complex, so that $R$ admits a canonical module $K$, say. Then, for each $\mathfrak{p} \in \operatorname{Spec}(R)$,

$$
E(R / \mathfrak{p}) \cong\left(V_{\mathrm{htp}} \times(R \backslash \mathfrak{p})\right)^{-\mathrm{htp}-1} K
$$

where $V_{r}$ is the triangular subset of $R^{r}$ defined in the paragraph just before the corollary.

Acknowledgment. I thank M. Tousi for his comment on 2.3. I also thank the referee for the invaluable comments on the manuscript.

\section{REFERENCES}

[A] Y. Aoyama, Some basic results on canonical modules, J. Math. Kyoto Univ. 23 (1983), 85-94.

[AG] Y. Aoyama And S. Goto, On the endomorphism ring of the canonical module, J. Math. Kyoto Univ. 25 (1985), 21-30.

[B-ZS] M. H. Bijan-Zadeh And R. Y. Sharp, On Grothendieck's local duality theorem, Math. Proc. Cambridge Philos. Soc. 85 (1979), 431-437.

[BH] W. Bruns and J. Herzog, Cohen-Macaulay Rings, Cambridge University Press, 1996.

[DT1] M. T. Dibaei And M. Tousi, The structure of dualizing complex for a ring which is $\left(S_{2}\right)$, J. Math. Kyoto Univ. 38 (1998), 503-516.

[DT2] M. T. Dibaei And M. Tousi, A generalization of dualizing complex structure and its applications, J. Pure and Applied Algebra, 155 (2001), 17-28.

[FFGR] R. Fossum, H.-B. Foxby, P. Griffith, and I. Reiten, Minimal injective resolutions with applications to dualizing modules and Gorenstein modules, Inst. Hautes Etudes Sci. Publ. Math., 45 (1976), 193-215.

[H] J. E. Hall, Fundamental dualizing complexes for commutative Noetherian rings, Quart. J. Math. Oxford 165 (1979), 21-32.

[HK] J. Herzog and E. Kunz, Der Kaninische Modul eines Cohen-Macaulay Rings, Lecture Notes Math. 238, Springer-Verlag, 1971.

[K] T. KaWASAKI, Finiteness of Cousin homologies, preprint.

[M] H. Matsumura, Commutative ring theory, Cambridge University Press, 1992.

[O] L. O'CARrol, On the generalized fractions of Sharp and Zakeri, J. Lodon Math. Soc. 28 (1983) 417-427.

[P] H. Petzl, Cousin complexes and flat ring extentions, Comm. Algebra, 25 (1997), 311-339. 
[ScTC] P. Schenzel, N. V. Trung and N. T. Cuong, Verallgemeinerte Cohen-MacaulayModuln, Math. Nachr. 85 (1978), 57-73.

[S1] R. Y. Sharp, The Cousin complex for a module over a commutative Noetherian ring, Math. Z., 112 (1969), 340-356.

[S2] R. Y. ShaRP, Gorenstein modules, Math. Z. 115 (1970), 117-139.

[S3] R. Y. Sharp, Finitely generated modules of finite injective dimension over certain CohenMacualay rings, London math. Soc. 25 (1972), 303-328.

[S4] R. Y. Sharp, Dualizing complexes for commutative Noetherian ring, Math. Proc. Cambridge Philos. Soc. 78 (1975), 369-386.

[S5] R. Y. Sharp, Local cohomology and the Cousin complex for a commutative Noetherian ring, Math. Z., 153 (1977), 19-22.

[S6] R. Y. Sharp, A commutative Noetherian ring which possesses a dualizing complex is acceptable, Math. Proc. Camb. Philos. Soc. 82 (1977), 197-213.

[SSc] R. Y. Sharp and P. Schenzel, Cousin complex and generalized Hughes complexes, Proc. London Math. Soc., 68 (1994), 499-517.

[SZ] R. Y. Sharp AND H. ZAKeri, Generalized fractions, Buchsbaum modules and generalized Cohen-Macaulay modules, Math. Proc. Cambridge Philos. Soc., 98 (1985), 429-436.

[T] N. V. Trung, Toward a theory of generalized Cohen-Macauly modules, Nagoya Math. J. 102 (1986), 1-49.

Institute for Studies in Theoretical Physics and Mathematics, P.O.Box 193955746, TEHran, Iran. 